\title{
EXTREMELY RED RADIO GALAXIES
}

\author{
Chris J. Willott, Steve Rawlings, Katherine M. Blundell \\ Astrophysics, University of Oxford, UK
}

\begin{abstract}
At least half the radio galaxies at $z>1$ in the 7C Redshift Survey have extremely red colours $(R-K>5)$, consistent with stellar populations which formed at high redshift $(z \gtrsim 5)$. We discuss the implications of this for the evolution of massive galaxies in general and for the fraction of near-IR-selected EROs which host AGN, a result which is now being tested by deep, hard X-ray surveys. The conclusion is that many massive galaxies undergo at least two active phases: one at $z \sim 5$ when the black hole and stellar bulge formed and another at $z \sim 1-2$ when activity is triggered by an event such as an interaction or merger.
\end{abstract}

\section{Introduction}

Radio sources are known to reside in massive, luminous host galaxies. Therefore, they are an excellent way of selecting such galaxies out to high redshifts and tracing their evolution. The obscuring torus, which forms the basis of the unified schemes, blocks the non-stellar nuclear emission from our line-of-sight providing a much clearer view of the host galaxy properties than is the case for quasars. $3 \mathrm{C}$ radio galaxies at $z \gtrsim 0.6$ have complex optical continuum and emission line structures aligned along their radio axes, which can generally be interpreted as due to recent (jet-induced) star-formation or non-thermal processes associated with the active nucleus (e.g. Best et al. 1998). Lacy et al. (1999a) have shown that the strength of the alignment effect decreases with decreasing radio luminosity such that fainter samples do not suffer from this problem.

The 7C Redshift Survey (7CRS) is a low-radio frequency $(151 \mathrm{MHz})$ flux-selected sample in three small patches of sky covering a total sky area of $0.022 \mathrm{sr}$. The flux-limit is $0.5 \mathrm{Jy}-$ a factor of $25 \times$ lower than the revised 3CR sample (Laing et al. 1983). We now have complete optical/near-infrared identifications for all the radio sources and $>90 \%$ spectroscopic redshifts. Further details of the survey are given in Willott 
et al. (2001a) and Lacy et al. (1999b). Only 7 out of 76 sources in the 7C-I and 7C-II regions lack spectroscopic redshifts. For these sources we have obtained optical and near-IR photometry in order to constrain their redshifts using photometric redshift techniques. We find that most of these seven galaxies have very red colours. A full account of this work is given in Willott et al. (2001b). A flat cosmology with parameters $\Omega_{\mathrm{M}}=0.3, \Omega_{\Lambda}=0.7$ and $H_{0}=70 \mathrm{~km} \mathrm{~s}^{-1} \mathrm{Mpc}^{-1}$ is assumed throughout.

\section{Results of photometric redshift fitting}

$R I J H K$ photometry was obtained for the seven sources without spectroscopic redshifts. Six of these objects have extremely red colours $(R-K>5.5)$, similar to those expected from evolved stellar populations at $z>1$. These data were fit with a range of instantaneous burst model galaxies (Bruzual \& Charlot in prep.) with redshift, age and reddening as free parameters. Best-fit models were found by searching for the minimum in the $\chi^{2}$ distribution. Fits with reduced $\chi^{2}<1$ were found for five objects and the remaining two had best-fit reduced $\chi^{2}<2$. An example of the SED-fitting is shown in Fig. 1.
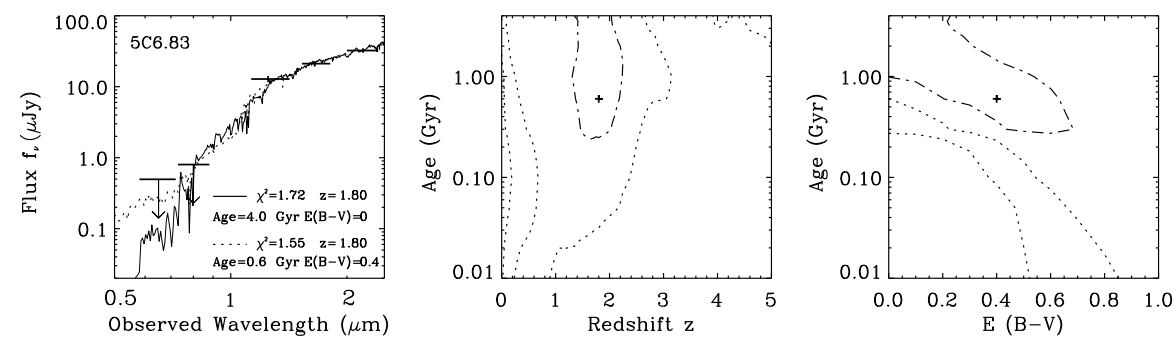

Figure 1. Example of model-fitting to the optical to near-IR SEDs of 7CRS radio galaxies. The left panel shows the broad-band photometric data of 5C6.83 $(7 \mathrm{CB} 021111.3+303948 ; R>24.5 ; K=18.3)$ with best-fit model galaxies. The best fit unreddened model is shown as a solid line and the best-fit reddened model as a dotted line. Details of these fits are given in the bottom-right corner. The centre and right panels show how the $\chi^{2}$ of the fit depends upon redshift, age of the stellar population and reddening. The minimum in reduced $\chi^{2}(1.55)$ is shown as a cross.

The best-fit redshifts for these radio galaxies ranges from $z=1.05$ to $z=2.35$ with typical uncertainties $\Delta z= \pm 0.3$. Considering other factors such as the $K-z$ relation and the lack of emission lines in their optical spectra, we believe that all the galaxies are likely to fall within the redshift range $1<z<2$. The flatness of the SEDs from $J$ to $K$ strongly argues against $z>2.5$. We know from near-IR spectroscopy that the colours are not strongly affected by emission line contamination, but in two cases there is a marginal emission line in the near-IR which 
could be $\mathrm{H} \alpha$ at $z \approx 1.5$, consistent with the SED-fitting. Quite a wide range of parameters provide acceptable fits for many of the galaxies. The degeneracy of age and reddening appears to be the strongest cause of this as shown by the diagonal shape of contours in the age-reddening plane in Fig. 1 (from old and dust-free at top-left to young and dusty at bottom-right). In contrast there is little correlation between redshift and age in the contours and the best-fit redshifts are in most cases similar for both the reddened and unreddened cases.

\section{The colours of $7 \mathrm{CRS}$ radio galaxies}

Since all members of the 7CRS (regions I and II) have been securely identified, have spectroscopic or photometric redshifts and have $R$ and $K$-band imaging (Willott et al. in prep), we can investigate the colour evolution of the radio galaxies. In Fig. 2 we plot the observed $R-K$ colour against redshift for the 49 narrow-line radio galaxies and the 2 broad-line radio galaxies (the 23 quasars are not plotted because their magnitudes are clearly dominated by non-stellar emission). The solid curve is a model featuring an instantaneous starburst at redshift $z=5$. At redshifts $z \leq 1$ the colours of most of the radio galaxies are close to the model curve suggesting that these colours result from very old galaxy populations with little (unobscured) current star-formation. Deviations to bluer colours can be caused by a small amount of more recent starformation or AGN-related processes like scattering of quasar light.

Moving to $z \gtrsim 1$ we find a marked increase in the scatter of the $R-K$ colours of the radio galaxies. This is probably due to a combination of two effects. First, at redshifts higher than $z \sim 0.8$, the observed $R$ band samples the rest-frame light below the $4000 \AA$ break. The ratio of fluxes below and above $4000 \AA$ is a very strong function of the amount of current or recent star-formation. Therefore small differences in the amount of recent star-formation will have a more dramatic effect on the observed colour at $z>0.8$. Lilly \& Longair (1984) showed that the optical/near-IR colours of 3CR galaxies at $z>1$ are inconsistent with a no-evolution model. Their observed $R-K \approx 4$ colours are similar to those of the bluest $7 \mathrm{CRS}$ radio galaxies. For the $3 \mathrm{C}$ objects these colours are best explained by recent star-formation in a few cases (Chambers \& McCarthy 1990), but in most cases, as further evidenced by large optical polarizations (e.g. di Serego Alighieri et al. 1989), they are probably caused by an extra non-stellar rest-frame UV component, typically scattered light from the quasar nucleus (see Best et al. 1998).

It is clear from Fig. 2 that, at $z>1$, the bluer 7CRS galaxies are much more likely to have redshifts measured from optical spectroscopy. 


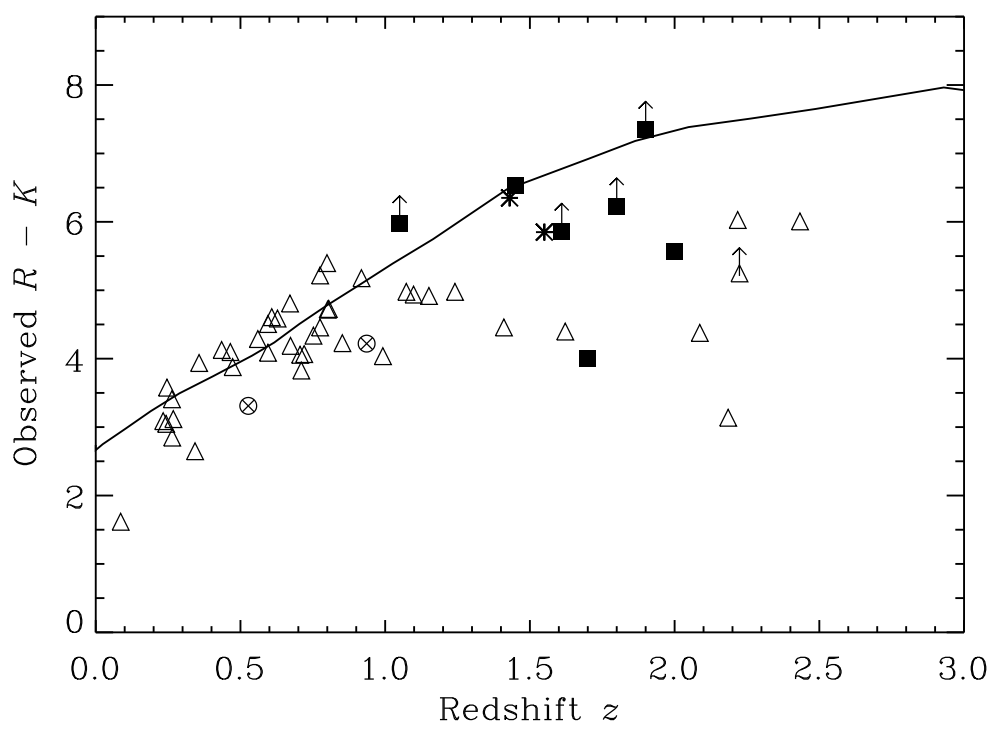

Figure 2. Observed $R-K$ colour as a function of redshift for radio galaxies in the 7C-I and 7C-II regions of the 7CRS. Triangles are narrow-line radio galaxies, crossed circles are broad-lined radio galaxies and filled squares are the seven 7CRS objects with photometric redshift estimates. The asterisks are the two red radio galaxies from the LBDS survey (Dunlop 1999). The solid line shows the evolution of the expected observed colour of a galaxy which formed in an instantaneous starburst at redshift $z=5$ using the models of Bruzual \& Charlot (in prep.). This model provides a good fit to the upper envelope of the galaxy colours up to at least redshift $z=2$.

This suggests that the bluer sources have stronger emission lines and are therefore good candidates for having non-stellar (AGN) and/or starburst components in the rest-frame UV. At $z>1$, radio galaxies from the $6 \mathrm{CE}$ sample, which has a flux-limit in between those of 3CRR and the 7CRS, also tend to have strong emission lines and blue colours (Rawlings et al. 2001). Therefore the reason that such a high fraction of extremely red radio galaxies at high-redshift has not been seen in previous complete samples is due to their higher radio flux-limits selecting only higher luminosity $z>1$ radio galaxies.

\section{Do the red colours indicate old galaxies?}

We have shown that at least half the $z>1$ radio galaxies in the 7CRS have red colours $(R-K>5)$, consistent with those expected from evolved stellar populations at these redshifts. In addition, the fact that the bluer high-redshift radio galaxies have stronger emission lines, suggests that they too may have underlying host galaxies which are very red. 
But can we be certain that these colours are an indicator of age, rather than the effects of reddening by dust? The answer is that we cannot be completely certain in the absence of high signal-to-noise spectroscopy of stellar absorption features. Due to the faintness of these galaxies in the optical ( $I \gtrsim 24)$, this will have to be performed in the near-infrared utilizing the low-background available with $\mathrm{OH}$-suppression spectrographs on large telescopes. Instead, we have to look at circumstantial evidence, such as that attained for similar objects. Dunlop et al. (1999 and references therein) have studied two red radio galaxies at $z \sim 1.5$ drawn from a faint radio sample. Deep Keck spectroscopy shows that the red colours of these galaxies are due to an old stellar population and not due to reddening by dust. The ages inferred are still controversial, although ages of $\sim 3$ Gyr remain the best estimate (Nolan et al. 2001). For these ages observed at $z=1.5$, the star-formation must have ceased at $z \sim 5$.

\section{Implications for AGN activity in elliptical galaxies}

Although powerful radio galaxies are very rare objects, we can use our findings to predict the relationship between other AGN (both radioloud and radio-quiet) and the near-infrared selected ERO population. Extrapolating down the radio luminosity function (Willott et al. 2001a) to less powerful radio galaxies, we find that if a similar fraction of these lower power radio galaxies have similar colours, they imply a space density of red radio galaxies which is about $3 \%$ of the space density of near-IR selected EROs (Daddi et al. 2000). Hence we find that a small, but significant, fraction $(\sim 3 \%)$ of field EROs are likely to be hosting radio-loud AGN.

However, it is well-known that luminous radio sources have limited lifetimes $\left(\sim 10^{8}\right.$ years $)$ which are much smaller than the Hubble time. The time elapsed between $z=2$ and $z=1$ is approximately $3.5 \mathrm{Gyr}$. Therefore if individual radio sources have lifetimes of only $\sim 10^{8}$ years, then the number of galaxies undergoing radio activity during this period would be a factor of 30 greater than that observed. Hence all of the near-IR selected EROs could plausibly undergo such a period of radio activity. A caveat to this is that the typical lifetimes of weak radio sources such as those which would dominate the ERO population are not well-constrained and could have longer lifetimes of $\sim 1$ Gyr. In such a case, only $\sim 10 \%$ of high- $z$ EROs would undergo a period of radio activity at some point (see Willott et al. 2001b for more details).

The hardness of the X-ray background requires that the space density of optically-obscured quasars exceeds that of optically-luminous quasars

(e.g. Comastri et al. 1995), which in turn are well known to outnumber 
radio-loud quasars by at least an order of magnitude. Many of the hard $\mathrm{X}$-ray sources discovered in Chandra surveys have very red galaxy counterparts with weak or absent emission lines (Crawford et al. 2001; Cowie et al. 2001). These objects are likely to be the radio-quiet analogues of the 7CRS EROs discussed here. The hard X-ray properties of the ERO population will be investigated with XMM-Newton and Chandra surveys of ERO fields. In the HDF-North Caltech area, 4 out of 33 EROs $(R-K>5)$ have hard X-ray detections (Hornschemeier et al 2001). Assuming an elliptical galaxy fraction in the field ERO population of $70 \%$ (Moriondo et al. 2001; Stiavelli \& Treu 2000) this corresponds to $\sim 20 \%$ of these ellipticals being observed to undergo a phase of AGN activity. Better statistics will accurately determine the duty cycle of AGN-activity in massive galaxies and provide constraints on quasar lifetimes (which are currently not well-constrained for radio-quiet quasars). It seems likely that rather than being an oddity, AGN activity is common in massive galaxies, not only during the high-redshift formation epoch, but also at a later stage once the major episode of star (and black hole) formation has long since ceased.

\section{References}

Best P.N., Longair M.S., Röttgering H.J.A., 1998, MNRAS, 295, 549

Chambers K.C., McCarthy P.J., 1990, ApJ, 354L, 9

Comastri A., Setti G., Zamorani G., Hasinger G., 1995, A\&A, 296, 1

Cowie L.L., et al., 2001, ApJL, in press, astro-ph/0102306

Crawford C.S., Fabian A.C., Gandhi P., Wilman R.J., Johnstone R.M., 2001, MNRAS, submitted, astro-ph/0005242

Daddi E., et al., 2000, A\&A, 361, 535

di Serego Alighieri S., Fosbury R.A.E., Tadhunter C.N., Quinn P.J., 1989, Nature, 341,307

Dunlop J.S., 1999, in The Most Distant Radio Galaxies, ed. P.N. Best, H.J.A. Röttgering, M.D. Lehnert, (KNAW Colloq.; Dordrecht: Kluwer), 14

Hornschemeier A.E., et al., 2001, ApJ, in press, astro-ph/0101494

Lacy M., Ridgway S.E., Wold M., Lilje P.B., Rawlings S., 1999a, MNRAS, 307, 420

Lacy M., et al., 1999b, MNRAS, 308, 1096

Laing R.A., Riley J.M., Longair M.S., 1983, MNRAS, 204, 151

Lilly S.J., Longair M.S., 1984, MNRAS, 211, 833

Moriondo G., Cimatti A., Daddi E., 2001, A\&A, in press, astro-ph/0010335

Nolan L.A., Dunlop J.S., Jimenez R., Heavens A.F., 2001, MNRAS, submitted, astro$\mathrm{ph} / 0103450$

Rawlings S., Eales S.A., Lacy M., 2001, MNRAS, 322, 523

Stiavelli M., Treu T., 2000, To appear in the proceedings of the conference "Galaxy Disks and Disk Galaxies", ASP Conf. series, eds. Funes and Corsini, astro-ph/0010100

Willott C.J., Rawlings S., Blundell K.M., Lacy M., Eales S.A., 2001a, MNRAS, 322, 536

Willott C.J., Rawlings S., Blundell K.M., 2001b, MNRAS, in press, astro-ph/0011082 